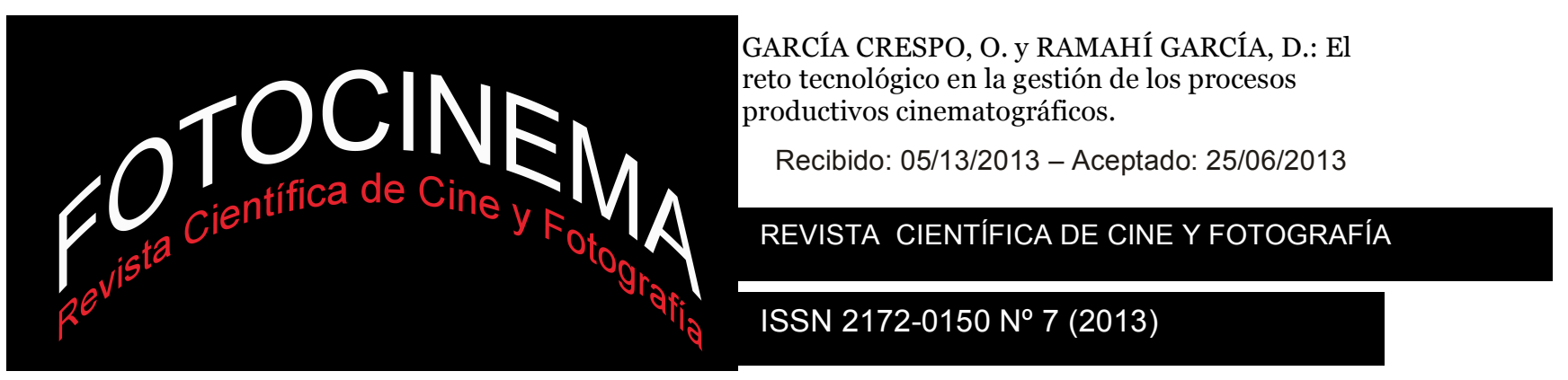

\title{
EL RETO TECNOLÓGICO EN LA GESTIÓN DE LOS PROCESOS PRODUCTIVOS CINEMATOGRÁFICOS; EL DIRECTOR DE POSTPRODUCCIÓN COMO EJE PARA LA IMPLEMENTACIÓN DE EFECTOS VISUALES EN ESPAÑA (FINALES DE LOS 90 - MAYO DEL 2012)
}

\section{THE TECHNOLOGICAL CHALLENGE IN THE CINEMATOGRAPHIC WORKFLOW MANAGEMENT: THE DIRECTOR OF POST PRODUCTION FOR THE IMPLEMENTATION OF VISUAL EFFECTS IN SPAIN (LATE 90'S - MAY 2012)}

\author{
Oswaldo García Crespo \\ Universidad de Vigo. España \\ Diana Ramahí García \\ Universidad de Vigo. España
}

\begin{abstract}
Resumen:
Este trabajo sobre la industria cinematográfica española se enmarca dentro de los estudios dedicados a analizar las consecuencias de la digitalización sobre la configuración de los procesos productivos y las competencias de los profesionales implicados. La postproducción cinematográfica en España es ya digital en la mayoría de los casos, culminando un proceso que se inicia a finales de los 90. Este hecho ha facilitado la digitalización de todos los flujos de trabajo y un proceso de traslación de parte de la creación de la imagen cinematográfica del rodaje a la postproducción. En este contexto estudiamos las implicaciones que se derivan de la normalización de los efectos visuales digitales como herramienta de producción para películas de alto y bajo presupuesto. Así, unido a las necesidades de producción de estos efectos, la volatilidad de la tecnología digital demanda una actualización constante en la dirección de producción del film y nos lleva a evaluar la importancia del director de postproducción como eje para la implementación de las
\end{abstract}

\section{Abstract:}

This paper deals with Spanish cinematographic industry and it belongs to the field of studies focused on the workflows digitalization configuration and the changes in the professional competences involved. Cinematographic postproduction in Spain is now digital in most of the cases, completing a process which begins at the end of the 90's. This fact has contributed to the digitalization of the whole production process and the translation of cinematographic image creation from the shooting to the postproduction. In this framework we approach the implications caused by the normalization of digital visual effects as production tool for high and low budget movies. Therefore, visual effects production needs together with ongoing evolution of digital technology require continuous updating for producers and lead us to assess the relevance of the director of postproduction as a key factor for the implementation of new workflows.

\section{Palabras clave:}

Digitalización; Postproducción; Efectos visuales; Director de postproducción.

\section{Keywords:}

Digitalization; Post production; Visual effects; Director of post production. 


\section{Tecnología digital, efectos visuales y producción cinematográfica}

Estamos acostumbrados a expresiones como "cine de acción”, “cine de Bergman" o "cine italiano". Estas locuciones se refieren al cine como manifestación artística. Sin embargo el adjetivo "digital" se refiere a la naturaleza tecnológica de las herramientas con las que ha sido producido, distribuido o exhibido. Este hecho llama la atención sobre la trascendencia de la implementación de la tecnología digital en el cine y sus consecuencias en la forma de producir películas. A pesar de ello, no debemos olvidar que una buena parte de las actuales rutinas de producción son una herencia del cine anterior a la aparición de la tecnología digital. Así, la gran mayoría de los términos son heredados de esa época y la filosofía que inspira las nuevas técnicas es en ocasiones la misma ${ }^{1}$. Además, la complejidad técnica es un factor que lleva condicionando y acompañando al cine desde sus inicios y que debemos valorar en relación al gran riesgo económico que suele acompañar los proyectos cinematográficos, en comparación con otros medios análogos en lo tecnológico, como la televisión o la publicidad².

La aplicación de la tecnología digital a la industria cinematográfica ha supuesto la introducción de la multiplicidad de formatos y de software, la introducción de flujos de trabajo en rodaje basados en la utilización de dispositivos de almacenamiento digital, la implementación de sistemas de producción virtual3, así como la democratización de la producción de ficción por el abaratamiento de los costes, los sistemas de trabajo en red, la piratería y un aumento progresivo en uso de efectos visuales digitales en el cine y la televisión (Honthaner, 2010, p. XV). En la historia de los efectos visuales, el cine de animación y el cine publicitario han jugado un papel esencial, fundamentalmente en lo que se refiere al avance de la tecnología asociada a

\footnotetext{
${ }^{1}$ Gross y Ward (2004, p. 12) advierten que: “[t]oday's moviemakers need to be aware of the past model to better understand their own duties and functions".

${ }^{2}$ Para Fernández y Martínez (1996, p. 21): “[es] el cine, sin duda, la referencia histórica de los modelos actuales de producción audiovisual para la difusión masiva. Su carácter pionero en la historia de los medios audiovisuales lo ha hecho antecesor directo y laboratorio de ensayo cuyas soluciones se han aplicado también a la industria del vídeo y de la televisión".

${ }^{3}$ Sistemas de planificación basados en entornos interactivos de previsualización (Previs).
} 
estos procesos ${ }^{4}$. Sin embargo, si bien son medios análogos en lo tecnológico, sus rutinas productivas y condicionantes industriales son específicos en cada caso, por lo que a partir de este punto nos referiremos a la denominada, de forma genérica, producción cinematográfica de ficción.

Así, el potencial de manipulación de los planos digitales en postproducción está provocando una nueva conceptualización de la imagen cinematográfica hasta el punto de que el material registrado por la cámara empieza a ser considerado un elemento visual más del plano final o material en bruto para su posterior composición (Manovich, 2011, p. 254). Este nuevo escenario implica que la tecnología haya creado nuevas formas de trabajo sometidas a una constante evolución tecnológica que provocan que los procesos de postproducción sean más complicados que nunca.

Ambas cuestiones, renovación tecnológica y modificación de las rutinas de producción, implican la necesidad por parte del equipo de producción de avanzar en el conocimiento de las técnicas digitales, en la medida en que van a influir en el criterio de elección de proyectos, elaboración de presupuestos o calendarios de trabajo. En última instancia, la digitalización de la producción de efectos visuales en concreto y de la fase de postproducción en general, están provocando un solapamiento de los flujos de trabajo, rompiendo con la linealidad de la producción cinematográfica: “[t]here was production and then there was postproduction. But that doesn't exist anymore. With digital post-production, you can do just about anything to that image that you want to without loss of quality” (Lucas, 2011, p. 8).

En el ámbito académico, la figura del productor cinematográfico ha sido objeto de simplificación en cuanto a sus competencias, hasta el punto de que la reiteración de análisis de carácter superficial ha llevado en muchos casos a la falta de definición de esta figura clave (Jacoste, 1996, p. 7). Cabe destacar

\footnotetext{
${ }^{4}$ Resulta interesante en este sentido el trabajo de Vidal (2008), que centra su tesis doctoral específicamente en la contribución del cine de animación a los efectos visuales y especiales en el cine. Asimismo el documental de Iwerks (2007) establece una clara relación entre los avances tecnológicos desarrollados por Pixar y las técnicas de composición digital y creación de elementos CGI que se utilizan en la actualidad. Ambas cuestiones, composición y elementos CGI, son pilares básicos en la producción de efectos visuales.
} 
una patente falta de trabajos de análisis y estudios en torno a la figura del productor y su equipo como eje para el desarrollo de los procesos productivos cinematográficos5. En el contexto profesional, la debilidad estructural del sector español ${ }^{6}$ parece influir negativamente en la proliferación de profesionales eficientes en este campo, lo que nos lleva a pensar que: “...las Facultades de Ciencias de la Comunicación de nuestro país, en especial del ámbito de la comunicación Audiovisual, deben tener entre sus objetivos la formación de buenos profesionales en este campo (Marzal y Gómez, 2009, p. 9).

Además de su intrínseco y legítimo carácter investigador y de reflexión, cabe situar este trabajo en un contexto estratégico en el que la Universidad se acerca a la realidad social y empresarial, en consonancia con algunas de las ideas que han inspirado el EEES7.

\section{Metodología}

Este artículo se centra en las consecuencias que la digitalización de los efectos visuales tiene sobre su inclusión en los planes de producción de películas españolas y cómo este hecho puede estar influyendo en la gestión de los procesos productivos. En última instancia trata de establecer una relación entre la tecnología digital y la necesidad de especialización en el núcleo de producción del film. Para ello tendrá en consideración el proceso de digitalización de la postproducción cinematográfica en España, que abarca desde finales de los años noventa a mayo del 2012, fecha de finalización del trabajo de campo realizado.

\footnotetext{
${ }^{5}$ Marzal y Gómez (2009, p. 9) aluden explícitamente a este vacío académico. Fernández y Martínez (1996, p. 21), trece años antes de la publicación anterior, ya habían detectado esta flaqueza: "[a]penas se ha escrito sobre las tareas de planificación y sobre los directores de producción implicados en la creación, distribución y explotación de los productos audiovisuales".

${ }^{6}$ En el año 2010 el 79\% de las productoras españolas participaron en una sola película. En años anteriores el porcentaje varía entre el 75\% y el 82\% (ICAA, 2011).

${ }^{7}$ En este contexto para Marzal y Gómez (2009, p. 13) “...sólo mediante un contacto estrecho con el mundo empresarial audiovisual seremos capaces de hacer posible que la formación universitaria sea cada vez más eficiente".
} 
Con la intención de incrementar la operatividad del objetivo enunciado nos planteamos la siguiente hipótesis de investigación:

La aplicación de tecnología digital a la producción de efectos visuales conlleva la necesidad de un productor especializado en postproducción.

La estrategia metodológica desarrollada para abordar nuestro objeto de estudio e intentar dar respuesta a la hipótesis planteada dio lugar a una estructuración de la investigación en dos fases, que han sido acometidas de forma secuencial.

Fase 1. Evaluación de la trascendencia de los efectos visuales en los presupuestos de las películas españolas.

El objetivo fundamental de esta fase fue la de conocer en qué medida las películas españolas están incluyendo los efectos visuales en sus planes de producción e identificar tendencias en cuanto al reparto de recursos. Para ello, lo más útil hubiese sido analizar los presupuestos de las películas una vez estas producciones hubiesen terminado, pero acceder a esta información en España constituye un verdadero reto. Es un condicionante que otros investigadores con objetos de estudio similares como Riambau y Torreiro (2009, p. 81) no dudan en señalar como un verdadero obstáculo para centros de investigación y universidades.

Así, tomamos la decisión de aproximarnos a los datos suministrados por el ICAA, fuente de información cuantitativa de la mayoría de publicaciones referidas a la industria cinematográfica. Sin embargo, la falta de actualización de las fuentes mencionadas, que no incluyen datos del ejercicio 2011, limitaba en cierta forma la posibilidad de observar la influencia de una crisis económica que está teniendo consecuencias directas sobre la industria cinematográfica (García, 2012). En cualquier caso, de la revisión del boletín informativo del ICAA 2011 se extrajeron datos porcentuales que nos permitieron aproximarnos a los objetivos de la investigación en este punto.

Fase 2. Análisis de la relación entre efectos visuales digitales y especialización en el núcleo de producción del film 
Esta fase se orientó a determinar los cambios que un aumento en la producción de efectos visuales digitales pudiera estar provocando en la forma de gestionar los recursos de un film.

Tras una fase de documentación y búsqueda de fuentes bibliográficas pudimos constatar la escasez de trabajos académicos sobre producción de efectos visuales en España. Esta etapa de la investigación estuvo condicionada además por el hecho de que la mayoría de estos estudios están desactualizados. Sí fueron útiles, sin embargo, fuentes de origen anglosajón que, sin embargo, resultaron ineficientes a la hora de abordar la especificidad de la industria cinematográfica española. Necesitábamos por tanto de una metodología complementaria, con capacidad de adaptarse a un objeto de estudio con un alto componente tecnológico y por lo tanto en constante evolución.

Procedimos por tanto a la revisión de investigaciones similares con la intención de identificar las herramientas metodológicas empleadas y valorar su aplicación a nuestro estudio. Así, el estudio "Organizational Creativity in Heterarchies: The Case of VFX Production” (Spelthann y Haunschild, 2011) tenía como objetivo analizar los contextos de creación colectiva que se dan en la producción de efectos viduales. Su interés por lo procesos productivos les llevó a justificar la realización de entrevistas semiestructuradas como estrategia metodológica básica para su estudio. Sin embargo los objetivos de este estudio no estaban asociados a una industria cinematográfica en concreto, lo cual constituye una característica importante de nuestra investigación. Desde este punto de vista el trabajo de Ciller (2009) resultaba una referencia más sólida, en la medida en que además de su interés por aproximarse a los procesos productivos desde el punto de vista de la gestión de la producción, acotaba su objeto de estudio a la industria cinematográfica española. También en este caso la autora optaba por la realización de entrevistas personales con profesionales asociados a la fase de postproducción. Por último, tuvimos también en cuenta la recomendación de 
Jacoste (1996, p. 184) que considera fundamental la inclusión de los profesionales del medio en investigaciones de este tipo.

La entrevista como solución metodológica en el ámbito de las Ciencias Sociales constituye una herramienta que De Miguel (2005, p. 252) vincula al cumplimiento de cuatro objetivos principales: obtención de información acerca de las entidades comunicativas, facilitar el análisis de las relaciones existentes entre estas entidades, conocer aspectos de estas relaciones opacos a través de otros métodos de investigación científica y ejercer un efecto clarificador en los procesos de inducción y deducción analíticos. Teniendo en cuenta estos objetivos, pusimos en marcha un trabajo de campo basado en el diseño de un panel de profesionales con la intención de realizar entrevistas estructuradas basadas en un cuestionario estándar, que incluimos a continuación.

\begin{tabular}{|c|c|c|}
\hline \multicolumn{3}{|c|}{ Modelo de cuestionario } \\
\hline \multicolumn{3}{|c|}{ Nombre } \\
\hline \multicolumn{3}{|c|}{ Perfil profesional } \\
\hline \multicolumn{3}{|c|}{$\begin{array}{l}\text { Empresa a la que } \\
\text { pertenece }\end{array}$} \\
\hline \multicolumn{3}{|c|}{ Formación } \\
\hline \multicolumn{3}{|c|}{ Años de experiencia } \\
\hline \multicolumn{3}{|c|}{ Fecha de la entrevista } \\
\hline \multicolumn{3}{|r|}{ Introducción y cuestiones } \\
\hline \multicolumn{3}{|c|}{$\begin{array}{l}\text { Las siguientes preguntas forman parte de un estudio académico sobre producción } \\
\text { cinematográfica en el ámbito español, por lo que aquellas cuestiones que se } \\
\text { relacionen con la industria cinematográfica, se referirán específicamente a este } \\
\text { ámbito. }\end{array}$} \\
\hline 1 & $\begin{array}{l}\text { ¿ En qué medida } \\
\text { creciendo en rela }\end{array}$ & $\begin{array}{l}\text { ecursos destinados a la producción de efectos visuales está } \\
\text { a los medios destinados a la fase de rodaje? }\end{array}$ \\
\hline 2 & $\begin{array}{l}\text { ¿Hasta qué punto } \\
\text { son gestionados e }\end{array}$ & $\begin{array}{l}\text { procesos productivos asociados a la fase de postproducción } \\
\text { ras fases de la producción cinematográfica? }\end{array}$ \\
\hline 3 & $\begin{array}{l}\text { ¿Cómo piensa qu } \\
\text { en la fase de roda }\end{array}$ & $\begin{array}{l}\text { procesos de producción de efectos visuales pueden influir } \\
\text { una película? }\end{array}$ \\
\hline 4 & ¿Cómo evalúa la u & ación de efectos visuales digitales como herramienta de \\
\hline
\end{tabular}




\begin{tabular}{|l|l|l|}
\hline & \multicolumn{2}{|l|}{ producción para aumentar la eficiencia de un rodaje? } \\
\hline 5 & $\begin{array}{l}\text { ¿De qué forma un aumento en la producción de efectos visuales puede afectar a } \\
\text { los equipos de producción y dirección? }\end{array}$ \\
\hline 6 & $\begin{array}{l}\text { La democratización en el } \\
\text { acceso a la herramientas } \\
\text { digitales para la producción de } \\
\text { efectos visuales... }\end{array}$ & \begin{tabular}{l} 
¿Implica un aumento en la cantidad de \\
profesionales competitivos en ese ámbito? \\
\cline { 2 - 2 } procesos productivos de las películas \\
nacionales, favorece el intrusismo o ambas \\
cosas?
\end{tabular} \\
\hline 7 & $\begin{array}{l}\text { ¿Cuáles son los aspectos más importantes a tener en cuenta para la selección de } \\
\text { los medios técnicos y humanos implicados en la producción de efectos visuales? }\end{array}$ \\
\hline 8 & $\begin{array}{l}\text { ¿Qué perfiles especializados en postproducción digital son demandados para la } \\
\text { nueva producción cinematográfica? }\end{array}$ \\
\hline 9 & $\begin{array}{l}\text { ¿Cómo afecta la digitalización creciente de los procesos productivos en la } \\
\text { industria cinematográfica a las competencias del productor? }\end{array}$ \\
\hline 10 & $\begin{array}{l}\text { ¿Cómo puede afectar la digitalización creciente de los procesos productivos en la } \\
\text { industria cinematográfica a las competencias de los equipos de dirección y de } \\
\text { producción? }\end{array}$ \\
\hline 11 & $\begin{array}{l}\text { ¿Cuáles son los retos que la producción de efectos visuales debe resolver en un } \\
\text { futuro en términos de eficiencia? }\end{array}$ \\
\hline 12 & $\begin{array}{l}\text { ¿Quiere añadir algo en relación a alguno de los temas tratados; procesos } \\
\text { productivos en postproducción, las competencias del productor en España, uso } \\
\text { de herramientas digitales...? }\end{array}$ \\
\hline Tabla 1. Fuente: Elaboración propia. \\
\hline
\end{tabular}

Este cuestionario se aplicó a profesionales del sector. Con la intención de hallar máximo el nivel elevado de representatividad de acuerdo a los objetivos planteados en la investigación tratamos de establecer criterios cuantitativos que relacionasen, como factor de inclusión en el panel de profesionales, la pertenencia a empresas del sector de la postproducción con mayor volumen de facturación. Así, se consultó la base de datos Sabi, de la que sólo constan datos actualizados a diciembre del 2010 y se extrajeron resultados parciales que a posteriori resultaron poco operativos. Por poner un ejemplo, Molinare, que presentó una de las cifras más altas del sector en 
ingresos de explotación ${ }^{8}$, presentó un ERE en el 2011, se fusionó con Infinia9 creando Imasblue y a finales de febrero del 2012 llevó a cabo otro ERE temporal ("Infinia (Landon) y Molinare (Mediapro) firman un ERTE cuando aún no se cumple un año de su ERE”, 2012). Como resultado, Ferrán Piquer, antiguo director del departamento de cine digital de Molinare y uno de los supervisores de efectos visuales más premiados en nuestro país se encuentra en la actualidad trabajando de freelance.

La actual coyuntura económica ha provocado que algunos profesionales con prestigio mantengan una relación accidental con muchas empresas, incapaces de afrontar sus salarios de forma continuada. Por otro lado, la polarización de los presupuestos de las películas nacionales obliga a tener en cuenta las diferencias existentes en la producción de efectos visuales entre las películas de alto y bajo presupuesto. El reducido tamaño de nuestro universo de estudio reduce el margen de error en este sentido, ya que obliga a las productoras de efectos visuales y profesionales independientes a afrontar tanto trabajos de bajo como de alto presupuesto para afrontar su supervivencia empresarial.

Por todo ello se plantearon una serie de criterios de carácter cualitativo que, combinados, pretendían conseguir un mayor grado de efectividad y que apuntamos de forma esquemática a continuación:

- Debían ser profesionales con una trayectoria lo suficientemente amplia como para haber asistido al proceso de digitalización de la fase de postproducción comenzado en España entre finales de la década de los 90 y principios de siglo XXI.

- Tendrían preferencia los perfiles profesionales que despiertan sinergias con los objetivos de esta investigación. Son dos; supervisores de efectos visuales e integrantes del equipo de producción. Además se incluirían profesionales con otros perfiles que nos permitan observar nuestro objeto de estudio desde el punto de vista de otros agentes implicados.

\footnotetext{
${ }^{8} 15$ millones de euros al final del ejercicio del 2010. En Bureau Van Dijk (s.f.).

${ }^{9} 14$ millones de ingresos de explotación en el ejercicio 2010. Ibíd.
} 
- Se consideraría que hayan participado en películas premiadas por la Academia del Cine ${ }^{10}$. Se valoró asimismo el éxito comercial y el que hayan recibido premios a su labor profesional.

- Se trataría de incluir profesionales que hayan participado en producciones que constituyan ejemplos paradigmáticos en cuanto al uso de efectos visuales en España.

- Se atendería a la polarización de presupuestos de las películas españolas incluyendo en el panel los responsables del equipo de producción de dos producciones con un elevado número de efectos visuales pero con presupuestos por encima y por debajo de la media.

Bajo estos criterios se elaboró un panel de profesionales adaptado a los objetivos de nuestra investigación.

\begin{tabular}{|c|c|c|c|}
\hline \multicolumn{4}{|c|}{ Panel de profesionales } \\
\hline Nombre & $\begin{array}{l}\text { Perfil } \\
\text { profesional }\end{array}$ & $\begin{array}{l}\text { Años de } \\
\text { experien } \\
\text { cia }\end{array}$ & $\begin{array}{l}\text { Sinergias con las hipótesis de esta } \\
\text { investigación }\end{array}$ \\
\hline Luis Manso & $\begin{array}{l}\text { Director de } \\
\text { producción }\end{array}$ & 20 & $\begin{array}{l}\text { Productor de películas paradigmáticas } \\
\text { en la historia de los efectos visuales en } \\
\text { España como El Milagro de P. Tinto, La } \\
\text { Gran Aventura de Mortadelo y Filemón } \\
\text { o Camino entre otras. Sus películas han } \\
\text { recibido varios premios Goya a "Mejores } \\
\text { efectos especiales", premio que él mismo } \\
\text { ha recibido en } 2003 \text { como director de } \\
\text { producción. }\end{array}$ \\
\hline $\begin{array}{l}\text { Sandra } \\
\text { Hermida }\end{array}$ & $\begin{array}{l}\text { Director de } \\
\text { producción }\end{array}$ & 20 & $\begin{array}{l}\text { Productora de La noche de los Girasoles } \\
\text { o Biutiful entre otras. Acaba de terminar } \\
\text { Lo Imposible, una película con una gran }\end{array}$ \\
\hline
\end{tabular}

${ }^{10}$ La Academia del Cine en España no diferencia, en el contexto de los premios Goya, las disciplinas de efectos especiales y visuales, quedando ambas encuadradas en la categoría de "Mejores efectos especiales".

52 


\begin{tabular}{|c|c|c|c|}
\hline & & & $\begin{array}{l}\text { cantidad de recursos dedicado a la } \\
\text { producción de efectos visuales. } \\
\text { Ganadora de un Goya por su trabajo en } \\
\text { El Orfanato. }\end{array}$ \\
\hline Xosé Zapata & $\begin{array}{l}\text { Director de } \\
\text { producción }\end{array}$ & 18 & 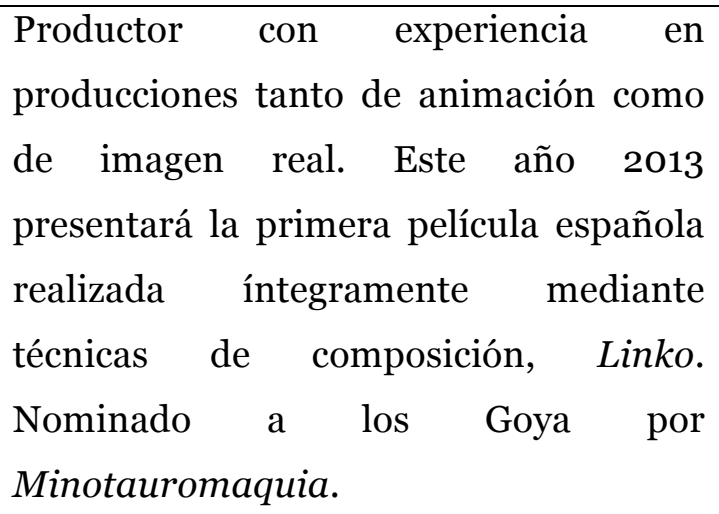 \\
\hline $\begin{array}{l}\text { Jaume } \\
\text { Martí }\end{array}$ & $\begin{array}{c}\text { Director de } \\
\text { postproducción }\end{array}$ & 13 & $\begin{array}{l}\text { Trabajó en películas como El truco del } \\
\text { Manco o Frágiles. Esta última es } \\
\text { ganadora del "Goya a mejores efectos } \\
\text { especiales". Su trayectoria profesional, } \\
\text { siempre vinculada a la fase de } \\
\text { postproducción, le ha permitido } \\
\text { convertirse en uno de los pocos } \\
\text { directores de postproducción del cine } \\
\text { español. }\end{array}$ \\
\hline $\begin{array}{l}\text { Marian } \\
\text { Briozzo }\end{array}$ & $\begin{array}{c}\text { Directora de } \\
\text { postproducción }\end{array}$ & 12 & $\begin{array}{l}\text { Es una de las directoras de } \\
\text { postproducción con más experiencia en } \\
\text { la industria española y ha participado en } \\
\text { películas como Slam, primera } \\
\text { producción española rodada en digital, y } \\
\text { en films con una gran cantidad de } \\
\text { efectos visuales como la premiada } \\
\text { internacionalmente Che, el Argentino o } \\
\text { la más reciente There Be Dragons. }\end{array}$ \\
\hline Félix Bergés & $\begin{array}{c}\text { Supervisor de } \\
\text { efectos } \\
\text { visuales }\end{array}$ & 20 & $\begin{array}{l}\text { Responsable de los efectos visuales en } \\
\text { películas como Ágora, Mar Adentro, } \\
\text { The Others, La Gran aventura de } \\
\text { Mortadelo y Filemón y tantas otras, por } \\
\text { los que ha recibido numerosos premios, }\end{array}$ \\
\hline
\end{tabular}




\begin{tabular}{|c|c|c|c|}
\hline & & & $\begin{array}{l}\text { entre ellos seis premios Goya. Es } \\
\text { considerado uno de los pioneros de los } \\
\text { efectos visuales en España. }\end{array}$ \\
\hline $\begin{array}{l}\text { Ferrán } \\
\text { Piquer }\end{array}$ & $\begin{array}{l}\text { Supervisor de } \\
\text { efectos } \\
\text { visuales }\end{array}$ & 24 & $\begin{array}{l}\text { Supervisor de efectos visuales en La } \\
\text { Chispa de la Vida, Amador o Balada } \\
\text { Triste de Trompeta, trabajo por le que } \\
\text { recibió un Goya. Es uno de los } \\
\text { supervisores de efectos visuales con más } \\
\text { experiencia en España. }\end{array}$ \\
\hline $\begin{array}{l}\text { José María } \\
\text { Aragonés }\end{array}$ & $\begin{array}{l}\text { Supervisor de } \\
\text { efectos } \\
\text { visuales }\end{array}$ & 21 & $\begin{array}{l}\text { Recibió un Goya por su trabajo en la } \\
\text { pionera Buñuel y la Mesa del Rey } \\
\text { Salomón y últimamente ha trabajado en } \\
\text { la premiada Chico y Rita. Dirige la } \\
\text { productora Apuntolapospo, que ha } \\
\text { formado parte del proyecto europeo de } \\
\text { investigación IMP, destinado a la mejora } \\
\text { del flujo de trabajo en postproducción. }\end{array}$ \\
\hline $\begin{array}{l}\text { Javier } \\
\text { García }\end{array}$ & $\begin{array}{l}\text { Supervisor de } \\
\text { efectos } \\
\text { visuales }\end{array}$ & 12 & $\begin{array}{l}\text { Supervisor de efectos visuales en Eva, } \\
\text { ganadora del "Goya a los mejores efectos } \\
\text { especiales". Recientemente ha trabajado } \\
\text { en Lo Imposible y dirige Fassman, una } \\
\text { de las productoras de efectos visuales } \\
\text { más activas de España en la actualidad. }\end{array}$ \\
\hline $\begin{array}{c}\text { Reyes } \\
\text { Abades }\end{array}$ & $\begin{array}{l}\text { Supervisor de } \\
\text { efectos } \\
\text { especiales }\end{array}$ & 44 & $\begin{array}{l}\text { Supervisor de efectos especiales desde } \\
\text { 1973. Ganador de nueve premios Goya } \\
\text { participando en películas de muy diversa } \\
\text { índole, desde iAy, Carmela! a Balada } \\
\text { triste de trompeta. }\end{array}$ \\
\hline $\begin{array}{c}\text { Raúl } \\
\text { Romanillos }\end{array}$ & $\begin{array}{l}\text { Supervisor de } \\
\text { efectos } \\
\text { especiales }\end{array}$ & 20 & $\begin{array}{l}\text { Ganador de } 5 \text { Goya por su trabajo en las } \\
\text { producciones: El milagro de P. Tinto, } \\
\text { Nadie conoce a nadie, La comunidad o } \\
\text { La gran aventura de Mortadelo y }\end{array}$ \\
\hline
\end{tabular}




\begin{tabular}{|c|c|c|c|}
\hline & & & Filemón. \\
\hline $\begin{array}{l}\text { Daniel } \\
\text { Monzón }\end{array}$ & Director & 20 & $\begin{array}{l}\text { Director de Celda } 211 \text { o La caja Kovak. } \\
\text { En la actualidad dirige El Niño, con una } \\
\text { alta carga de trabajo en efectos visuales. }\end{array}$ \\
\hline $\begin{array}{c}\text { Teo } \\
\text { Delgado }\end{array}$ & $\begin{array}{l}\text { Director de } \\
\text { fotografía }\end{array}$ & 23 & $\begin{array}{l}\text { Director de fotografía en Hola, ¿estás } \\
\text { sola?, Flores de Otro Mundo o Torrente } \\
\text { 4, su dilatada experiencia laboral y perfil } \\
\text { profesional le ha permitido asistir en } \\
\text { primera persona a la eclosión de los } \\
\text { efectos visuales digitales en España. }\end{array}$ \\
\hline
\end{tabular}

Además de constituir la columna vertebral de esta segunda fase de la investigación, los resultados de las entrevistas han servido de apoyo para la realización de otros trabajos centrados en la postproducción cinematográfica, así como han dado lugar a nuevas vías de investigación. Así, fuentes bibliográficas y digitales de ámbito anglosajón en su mayoría, unidas a un trabajo de campo basado en la realización de entrevistas estructuradas a profesionales del cine español, constituyen las herramientas básicas para afrontar los objetivos de este trabajo.

\section{Los efectos visuales en la industria cinematográfica española}

La tecnología digital ha permitido aumentar las posibilidades de manipulación de la imagen cinematográfica en postproducción para producciones de alto y bajo presupuesto en la industria cinematográfica española, por lo que casi cualquier película contiene alguna imagen generada por ordenador o alterada digitalmente ${ }^{11}$. La evolución tecnológica reciente ha permitido el acceso a la producción de efectos visuales a proyectos de bajo presupuesto que hace tan sólo unos años no hubieran podido asumir los

11 S. Hermida (entrevista personal, 15 de marzo, 2012) afirma que: "[e]s muy difícil encontrar una película donde no haya postproducción digital y efectos digitales de ningún tipo.(...) Aunque sea uno hecho en un Maya pirateado en un ordenador personal. Algo habrá. La digitalización no es una tendencia. Está ahí” 
costes derivados de su utilización ${ }^{12}$. Este hecho conlleva un gran impacto si tenemos en cuenta que el tejido empresarial cinematográfico español está formado en su mayor parte por este tipo de proyecto. Así, desde que esta manipulación sistemática va acompañada de una mayor accesibilidad a los sistemas de software y hardware dedicados a ello, podemos empezar a hablar de una generalización de la producción de efectos visuales como parte de la producción cinematográfica.

A través del trabajo de campo hemos constatado que, en la opinión de todos los profesionales entrevistados, se está produciendo un aumento proporcional de la partida de efectos visuales en los presupuestos de las películas españolas: "el porcentaje dedicado a efectos visuales de cada película hace unos años era cero y ahora en las películas tienes un 1\% o un 2\% y en las películas grandes pueda llegar a un 5, 6 y a veces hasta un 10\%” (Félix Bergés, entrevista personal, 23 de marzo, 2012). Por otro lado, la ausencia de una cultura de cesión documental por parte de las productoras cinematográficas a centros de investigación y estudio, nos obliga, para la realización de un análisis de carácter cuantitativo, a valernos de los datos proporcionados por el ICAA, que se derivan de presupuestos previos a la producción del film. Así, su modelo de presupuesto presenta una estructuración por capítulos y partidas que no contienen un apartado específico para la inclusión de gastos derivados de la utilización de efectos visuales, que acaban siendo incluidos en el capítulo 9, en la partida de varios (Sandra Hermida, entrevista personal, 15 de marzo, 2012). La consulta de esos datos nos ha permitido constatar que se está produciendo un aumento porcentual del capítulo 9, pero en este capítulo se incluyen otros gastos que tendrían que ser tenidos en consideración, y que están directamente relacionados con el uso del celuloide en postproducción. Sin embargo, el capítulo 8, dedicado al apartado fotoquímico, se reduce porcentualmente año

\footnotetext{
${ }^{12}$ Para el director de producción Luis Manso: “...el avance de la tecnología y la reducción de costes hace que realmente esos costes sean más aquilatados. Hoy las películas tienen más postproducción digital que hace unos años y sin embargo el coste proporcionalmente es menor. La importancia es creciente aunque en términos económicos cada vez esa importancia nos sale más barata." (L. Manso, entrevista personal, 24 de febrero, 2012).
} 
a año, lo que nos permite deducir que aquellas partidas del capítulo 9 dedicadas al tratamiento de formatos analógicos en postproducción no pueden estar aumentando en tanto en cuanto el uso de material fotoquímico en rodaje tiende a disminuir. Ello nos permite constatar un aumento porcentual de recursos para la producción de efectos visuales en España.

\begin{tabular}{|l|l|l|}
\hline \multicolumn{3}{|c|}{ Evolución del gastos por partidas presupuestarias } \\
\hline $\begin{array}{l}\text { Año 2oo8: Muestra de 26 } \\
\text { largometrajes }\end{array}$ & Capítulo 8 & Capítulo 9 \\
\hline Coste (MM) & $2.536 €$ & $3.668 €$ \\
\hline Coste medio & $98 €$ & $141 €$ \\
\hline \% Medio & 3,72 & 5,36 \\
\hline Año 2oo9: Muestra de 15 largometrajes & Capítulo 8 & Capítulo 9 \\
\hline Coste (MM) & $1.066 €$ & $3.289 €$ \\
\hline Coste medio & $71,06 €$ & $219,26 €$ \\
\hline \% Medio & 2,22 & 6,85 \\
\hline $\begin{array}{l}\text { Año 2o10: Muestra de } 25 \\
\text { largometrajes }\end{array}$ & Capítulo 8 & Capítulo 9 \\
\hline Coste (MM) & $1090,59 €$ & $638,66 €$ \\
\hline Coste medio & $255,46 €$ & $255,46 €$ \\
\hline \% Medio & 1.92 & 10,36 \\
\hline Tabla 4. Elaboración propia. Fuente: ICAA (2011). & \\
\hline
\end{tabular}

Si bien consideramos que esta evolución va a ser constante, es necesario tener en cuenta la disminución de los costes medios de los films nacionales, que estaría incluyendo en la capacidad por parte de las películas de bajo presupuesto de adaptarse a un nuevo escenario de producción con un alto componente tecnológico ${ }^{13}$. En este sentido, la producción de efectos visuales en España está más condicionada por el factor económico que por la

${ }^{13}$ Éste es un factor clave desde el punto de vista industrial; la asimilación de tecnologías y los costes por debajo de la línea, que en el caso español suponen un porcentaje muy alto del presupuesto total del film. De esta forma la estereoscopía no ha acabado de implementarse en nuestro país, incapaz de flexibilizar la financiación de sus proyectos (X. Zapata, entrevista personal, 26 de marzo, 2012). 
accesibilidad de la tecnología necesaria para llevarlos a cabo (Vidal, 2008, p. 261).

Así, atendiendo a la polarización de los costes de las películas españolas, las pocas producciones nacionales con presupuestos por encima de la media están favoreciendo el desarrollo de la producción de efectos visuales en España, en tanto en cuanto no pueden recurrir a empresas extranjeras por el elevado coste de su oferta. De esta forma, las propias condiciones del mercado están favoreciendo el fortalecimiento del sector, al tiempo que provocan una disminución del control sobre los procesos y el resultado final. "Lo que ocurre es que las empresas se ven en la situación de ir aprendiendo mientras que lo van haciendo. Es lo que ha pasado en Lo imposible; ni el Ranchito ni Félix habían hecho nunca un Tsunami. Esto genera inseguridad con respecto a cantidades de tiempo, formas de rodaje, presupuesto, entregas y recursos invertidos” (S. Hermida, entrevista personal, 15 de marzo, 2012).

En cualquier caso, las películas españolas con presupuestos elevados asumen la producción de efectos visuales para llevar a cabo proyectos en los que estos efectos forman parte de la historia. Por otro lado, en los proyectos de bajo presupuesto se ha generalizado el uso de efectos visuales destinados a pasar desapercibidos para el espectador, de forma que la duplicación de figurantes, los apoyos al maquillaje y vestuario, la utilización de fondos virtuales o la eliminación de elementos indeseados del plano, se constituyen como recursos de uso común, que contribuyen a enriquecer la estrategia de producción del film.

En cualquier caso, los efectos visuales en España son una parte de la producción cada vez más relevante, a la luz de los datos disponibles, las publicaciones específicas sobre el tema (Ciller, 2009, pp. 119-127) y la opinión de profesionales del equipo de producción. De acuerdo con como Jaume Martí: “[e]videntemente es obvio que cada vez más los recursos destinados a la postproducción o concretamente a la producción de efectos visuales esta aumentando en todas las producciones; sean pequeñas, medianas o grandes" (J. Martí, entrevista personal, 21 de marzo, 2012). 


\section{El componente tecnológico en la gestión de los procesos productivos}

La tecnología digital en postproducción se implantó mucho antes de que se generalizase la utilización de formatos digitales en grabación o en exhibición. Los costes derivados de esta situación significaron un escollo importante para la generalización del uso de efectos visuales en España ${ }^{14}$. Por ello, cuando fue más barato o igualmente competitivo postproducir digitalmente una película en lugar de en fotoquímico, la producción de efectos visuales y el Digital Intermediate se generalizan. El uso de herramientas digitales se extiende con gran rapidez en la medida en que se está convirtiendo, más que en un factor de eficiencia, en un elemento básico para mantener la competitividad del producto cinematográfico: "cada vez es más asequible a cualquier proceso. Ahora las películas más baratas son las digitales. Una película en formato tradicional cuesta mucho más cara que en formato digital” (T. Delgado, entrevista personal, 27 de febrero, 2012).

Swartz (2005, p. 2) señala dos factores principales por los cuales existe una tendencia a introducir la tecnología digital en la postproducción de la imagen cinematográfica. En primer término, el hecho de que en el contexto digital las imágenes pueden copiarse sin que exista un deterioro entre el original y la copia. En segundo lugar, la precisión que ofrece la tecnología digital en el tratamiento de las imágenes; tanto en la modificación del color y su aspecto en general como en cuanto a la mejora de los procesos de composición.

Así, los trabajos de producción de efectos visuales son rutinas flexibles que suelen incluir la aplicación de decenas de combinaciones de procedimientos basados en factores de carácter tecnológico. Además cada proyecto cinematográfico constituye un prototipo, por lo que los equipos de producción deben adaptar el sistema de trabajo a cada película de forma que les permita llevar a cabo los objetivos, asumiendo además, las limitaciones presupuestarias (Lara, 2005, p. 17). El hecho de que incluso cada plano

\footnotetext{
${ }^{14}$ Nos referimos a los costes derivados del escaneado del material filmado y su posterior impresión en el negativo final, con todas las complicaciones y condicionantes que este proceso analógico-digital-analógico conlleva.
} 
presente distintas necesidades obliga al conocimiento de la tecnología aplicable en cada caso, pues será un factor determinante para resolver la configuración de los flujos de trabajo⒌ En paralelo, cada fase de tratamiento de la imagen digital en postproducción implicará la elaboración de una implementación concreta de cada flujo de trabajo específico o pipeline ${ }^{16}$ (Towsend, 2010, p. 532).

El estudio para la optimización de los flujos de trabajo está relacionado con el conocimiento de las herramientas digitales que forman parte de ellos. Es más, la introducción de estas herramientas está cambiando, en algunos casos, la configuración de los procesos productivos de los que forman parte (Silverman, 2005, p. 15) ${ }^{17}$. Sin embargo, la proliferación de herramientas en este ámbito haría imposible su mera catalogación: “[t]here are too many tools and too many ways to use these tools for any kind of meaningful discusión to take place" (Brinkman, 2008, p. 368). Además, cada una de esas herramientas responde a una situación particular de forma que no existen reglas generales a propósito de su idoneidad, en la medida en que ésta dependerá de cada situación particular y la flexibilidad de la herramienta.

Esta complejidad técnica provoca una situación de exigencia a los equipos de producción que surge de la necesidad de afinar el conocimiento sobre aquellas soluciones que puedan optimizar los recursos técnicos y humanos disponibles (Silverman, 2005, p. 17). Todo ello cristaliza en el trabajo del productor cinematográfico, que en palabras de Marzal y Gómez (2009, p. 10): “...habrá de poseer un conocimiento exhaustivo sobre todas las profesiones que cualquier obra audiovisual involucra, sobre los procesos implicados y sobre las tecnologías utilizadas”.

\footnotetext{
${ }^{15}$ En el contexto de la producción cinematográfica, podemos definir como: “... a especific set of procedures and deliverables that defines a goal. The overaching workflow is the total production workflow. The goal is make a movie, and that necessitates a set of procedures that results in the deliverable: the film. (Bugaj, 2010, p. 784).

${ }^{16}$ Pipeline es definido por Novy (2010, p. 786), en el contexto de la producción cinematográfica como "la implementación de las especificaciones de un flujo de trabajo".

${ }^{17}$ Incluso en ocasiones, en el contexto de la aplicación de las herramientas digitales, la única manera de evaluar su idoneidad es su aplicación y la experiencia que se deriva de la misma (Billups, 2003, p. 51).
}

60 
En el ámbito europeo existe una tendencia a la especialización en el equipo de producción. Así y todo, aún estamos lejos de contar con una mayoría de productores con perfil de experto, debido a la ausencia de un tejido industrial que garantice una continuidad en la actividad de las productoras, facilitando el perfeccionamiento de los profesionales implicados (Jacoste, 1996, p. 15).

\subsection{La implementación de efectos visuales en la producción cinematográfica en España}

Existe una creencia generalizada y errónea que sitúa la gestión de los efectos visuales como una faceta más de la fase de postproducción, cuando éstos pueden establecer relaciones con los departamentos de desarrollo de vestuario, con cuestiones relativas a la planificación de cámara o con la dirección de arte en alguno de sus apartados. Por ello, la producción de efectos visuales debe empezar en las primeras fases del proyecto cinematográfico. Es importante, por tanto, que el departamento de producción integre al equipo de efectos visuales tan pronto como le sea posible, ya que le permitirá interactuar con otros departamentos para la consecución de los objetivos de cada plano en el que estén involucrados. Asimismo podrá alertar al productor y director de las consecuencias que tendrán sus decisiones en la planificación de la postproducción: “...the early involvement of the VFX Supervisor and VFX Producer can save the production substantial sums of money" (Finance y Zwerman, 2010, p. 37) ${ }^{18}$. Parece previsible que la carga de trabajo para la producción de efectos visuales sea cada vez mayor, por ello el equipo de efectos visuales debe realizar esfuerzos de coordinación también en postproducción $y$ especialmente con el equipo de montaje para ajustarse a los objetivos fijados en el calendario de trabajo. Todo ello, junto con otros factores que

\footnotetext{
${ }^{18}$ También para Smith (1986, pp. 35-36) la inclusión del equipo de efectos visuales en la fase de preproducción constituye una buena manera de conseguir más eficiencia en términos de tiempo y dinero.
}

61 
analizaremos con posterioridad implican que, para Gross y Ward (2004, p. 224): "[t]he visual effect process is changing the way movies are made".

Sin embargo, y aunque resulte paradójico, a día de hoy en España aún es habitual la ausencia de un productor especializado en postproducción. Incluso es una práctica habitual que una vez finalizado el rodaje, el director de producción desaparezca (S. Hermida, entrevista personal, 15 de marzo, 2012). Ello se debe a una percepción errónea sobre la postproducción, en tanto en cuanto es considerada como un contexto creativo rutinario, cuando en la actualidad y cada vez más, es una fase de gran transcendencia.

"El proceso de creación es más constante. Antes se ponían sobre la mesa discusiones artísticas hasta que llegaba el rodaje y ahí acababa todo. Hoy en día esa relación se intenta mantener hasta que tienes la copia acabada porque hasta ese día se están tomando decisiones importantes a nivel de dirección y de producción” (J. Martí, entrevista personal, 21 de marzo, 2012).

En este contexto creemos que es útil señalar la principal de las competencias del director de postproducción que es para Ciller (2009, p. 414): “...la de conocer y seleccionar todos los recursos técnicos, humanos y actividades que integran esta etapa”. Para ello debe atesorar un gran conocimiento de los aspectos tecnológicos que condicionan la postproducción de imagen y sonido, lo que debe permitirle la elaboración razonada de un calendario de trabajo, que hará cumplir asumiendo labores de coordinación. Ello implicará dar el visto bueno a numerosas decisiones creativas como cerrar definitivamente el montaje de imagen, realizar los doblajes, componer y grabar las músicas, realizar efectos de sala y digitales, mezclas finales y obtener la copia definitiva de la película. Y si bien las decisiones del director de postproducción están condicionadas por las órdenes del director, productor y jefe de producción, en algún momento puede verse obligado a ejercer un control de tipo creativo. Para Ciller (2009, p. 414): "este hecho no es nada habitual en nuestro país; realizar esta función requiere de algo más que conocer escrupulosamente la técnica”.

62 
Por otro lado, sería recomendable la existencia de un interlocutor que gestione los intereses del equipo de producción de efectos visuales. Ello se deriva de una serie de necesidades comunicativas que Sandra Hermida (entrevista personal, 15 de marzo, 2012), como directora de producción, valora como esenciales: “[da] igual si diseñas una película con 30 efectos digitales o 300, tú lo que necesitas es hacer una estructura piramidal de responsabilidad. No puedes tratar con cada uno de los 50 profesionales que va a hacer esos efectos".

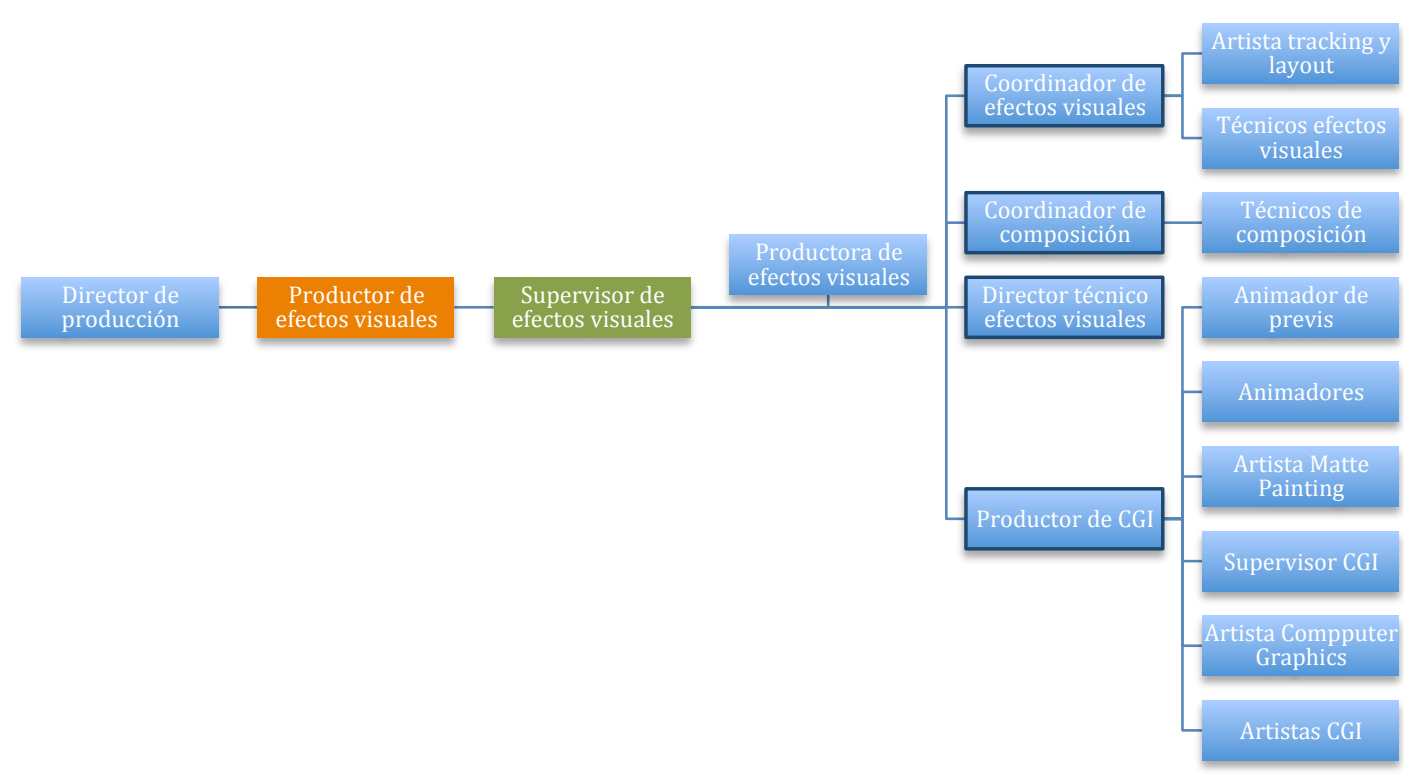

Fig. 6.1. Organigrama básico para la producción de efectos visuales en una película española de gran presupuesto. Fuente: Elaboración propia a partir de datos extraídos de las producción Ágora (Amenábar, 2009) y Eva (Maíllo, 2011).

El gráfico anterior dibuja una pirámide de responsabilidades que no encuentra su reflejo en el ámbito cinematográfico español. Una de las primeras cuestiones que debemos matizar tiene que ver precisamente con la ausencia del productor de efectos visuales ${ }^{19}$, incluso en aquellas que presentan una parte importante dedicada a la producción de estos efectos. Este hecho es analizado por Sandra Hermida (entrevista personal, 15 de marzo, 2012) que lo explica a través de dos factores. En primer lugar la

${ }^{19}$ El productor de efectos visuales es el responsable, según Finance y Zwerman (2010, p. 39) del departamento de efectos visuales. Así, su principal competencia será: “... look on the department as a businnes enterprise in wich the principal goal is bringing the visual effects in on Budget and on Schedule".

63 
existencia de un entramado industrial para la producción de efectos visuales con carácter accidental, basado en equipos freelance contratados por empresas diferentes. Y en segundo lugar, la falta de normalización de la existencia de un coordinador con capacidad de control y decisión sobre todas las empresas implicadas. A estas dos cuestiones debemos sumar otro factor: la mayoría de las producciones españolas presentan bajos presupuestos y un equipo humano muy limitado, lo que vendría a complicar una falta de adaptación en este sentido.

En el contexto cinematográfico español, las responsabilidades del productor de efectos visuales son asumidas por el director y el coordinador de postproducción si existiesen, por el supervisor de efectos visuales y por el departamento de producción de la productora de efectos visuales si el supervisor no es un freelance ${ }^{20}$. Y si bien el supervisor de efectos visuales es ya una figura habitual en el contexto cinematográfico español, no esta generalizada la existencia de un director ni la de un coordinador de postproducción, por lo que organigrama suele responder al segundo de los modelos detallados a continuación ${ }^{21}$.

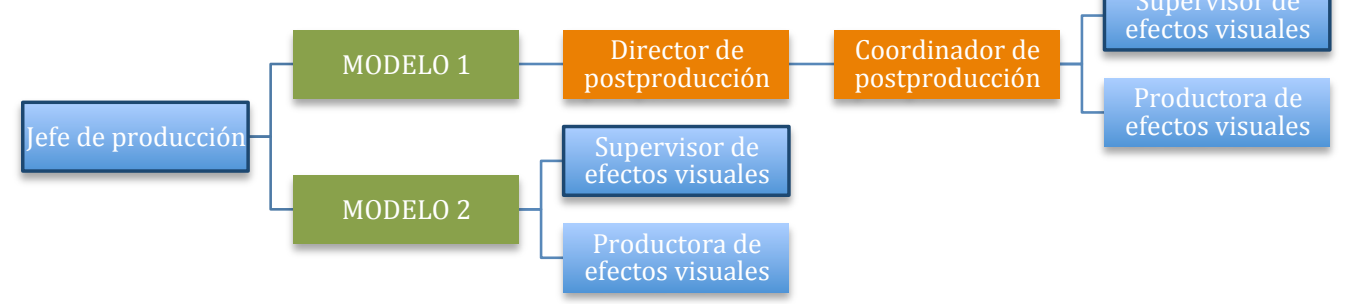

Fig. 6.2. Adaptación al contexto español del organigrama referente a la gestión de los recursos destinados a la producción de efectos visuales ${ }^{22}$. Fuente: Elaboración propia.

\footnotetext{
${ }^{20}$ Fue el caso de Ferrán Piquer en La Chispa de la Vida (De la Iglesia, 2011) (F. Piquer, entrevista personal, 1 de febrero, 2012).

${ }^{21} \mathrm{Al}$ igual que la figura del productor de efectos visuales, el director de postproducción debe abrirse camino al ejercer un determinado control artístico que en nuestro país, tradicionalmente, no va más allá de la esfera de actuación de productores y directores (Ciller, 2009, p. 122).

${ }^{22}$ Si bien el modelo 1 vendría a responder a producciones con un volumen elevado de recursos destinado a efectos visuales, el modelo 2 se corresponde con la gran mayoría de las producciones nacionales, en las que el supervisor de efectos visuales asume responsabilidades que corresponderían al núcleo de producción del film.
}

64 
Ante la inexistencia de un productor de efectos visuales y la habitual ausencia de un director de postproducción, las necesidades derivadas de la utilización de efectos visuales son implementadas bajo la responsabilidad del director de producción y el supervisor de efectos visuales, que actuará también como coordinador de la postproducción ${ }^{23}$. El supervisor de efectos tiene además una responsabilidad con respecto a los efectos visuales, análoga a la del director con respecto a la película en su conjunto. Debe saber realizar estimaciones acerca de las necesidades técnicas asociadas a un trabajo concreto y saber identificar las tareas de las que se compone cada trabajo, de analizar los resultados intermedios, de transmitir claramente las órdenes al equipo y de proporcionar soluciones de carácter creativo (Finance y Zwerman, 2010, p. 267). Esta aglomeración de funciones en el supervisor de efectos visuales podría estar provocando lagunas en la coordinación, principalmente en aquellos momentos en los que tenga que priorizar sus responsabilidades. Jaume Martí, como director de postproducción en The Impossible (Bayona, 2012) relata su experiencia en este sentido:

"El supervisor de efectos visuales estaba en rodaje diciendo cómo se tenían que rodar los planos que iban a formar parte de esos efectos y una vez que vamos a postproducción se mete en la sala y entrega planos, pero tiene que haber alguien que haga un seguimiento y haga listados de necesidades.... y eso lo hice yo. Hay desfase en el equipo de producción. No puede ser que en rodaje sean los que son y luego en postproducción seamos dos" (J. Martí, entrevista personal, 21 de marzo, 2012).

Los propios supervisores de efectos visuales, como Ferrán Piquer (entrevista personal, 1 de febrero, 2012), reivindican la figura del productor especializado en postproducción: “... hay un perfil muy necesario y que se le da poca importancia que es el productor técnico. Es difícil encontrar gente que entienda de todo tipo de postproducción que sea capaz de distribuir bien

${ }^{23}$ Para Ciller (2009, p. 122) el coordinador de postproducción se diferencia del director de postproducción en un mayor especialización técnica y en una ausencia de responsabilidad sobre el proceso creativo, limitándose únicamente a una labor de supervisión.

65 
ese trabajo y prepararlo ${ }^{24}$. La ausencia de un director de postproducción constituye una debilidad estructural asociada a la imposibilidad, por parte del director de producción, de tener un conocimiento tan específico de todos los procesos que le permita tomar la decisión más eficiente en cada caso (Javier García, entrevista personal, 12 de marzo, 2012). Por todo ello, parece razonable que las posibilidades de las herramientas digitales asociadas a la producción de efectos visuales obliguen a los equipos de producción en España a crear perfiles especializados que antes de la digitalización no existían.

\subsection{La necesidad de un productor experto: el director de postproducción}

Algunos directores de postproducción llevan desempeñando su labor desde los inicios de la digitalización de la postproducción. Desde entonces hasta ahora el número de profesionales que ocupan este cargo ha aumentado, si bien su presencia sigue siendo casi anecdótica.

En un contexto productivo condicionado por un aumento de la importancia de la fase de postproducción en la creación cinematográfica, cobra sentido que el director de postproducción coordine reuniones previas al rodaje junto con el director de producción, el director y el montador. El objetivo de estas reuniones entre otras cuestiones será optimizar la coordinación, lo que permitirá tomar decisiones que pueden beneficiar a la producción en términos de la calidad y eficiencia ${ }^{25}$. Ya durante la fase de rodaje será habitual que un profesional del equipo de efectos visuales participe

\footnotetext{
${ }^{24}$ Ferrán Piquer (entrevista personal, 1 de febrero, 2012), como supervisor de efectos visuales, asume con regularidad la labor de coordinador digital y afirma que cada vez se incorpora antes a al proyecto, influyendo sobre las estrategias necesarias para llevar a cabo la película. Todo ello está dando lugar nuevas vías de comunicación y nuevos contextos productivos: "[e]l departamento de decorado cada vez está más implicado con el de efectos visuales. El flujo de trabajo para la extensión digital de decorados implica una relación más estrecha con el departamento de arte que antes no se daba" (F. Piquer, entrevista personal, 1 de febrero, 2012).

${ }^{25}$ Estas reuniones de equipo tendrán su continuación, según la misma autora (Ciller, 2009, p. 122), durante la fase de postproducción.
}

66 
aportando su punto de vista en cuanto a la mejor manera de obtener el material que formará parte de los efectos previstos en el montaje final. La presencia del director de postproducción estaría articulando la creación de ese espacio necesario para que el supervisor de efectos visuales asegure el cumplimiento de los planes de producción tal y como se habían acordado (M. Briozzo, entrevista personal, 22 de marzo, 2012). Sin embargo, según algunos de los profesionales consultados y sin negar la tendencia a que estas situaciones se produzcan cada vez en mayor medida, a las películas españolas aún les queda un largo camino por recorrer en términos de optimización de los flujos de trabajo:

"En el 80\% de las producciones no tenemos una reunión entre los equipos de efectos digitales y tradicionales, no tenemos una lectura de guión y de necesidades, no tenemos un proyecto (...) Se consigue muy poco no juntando a las partes; producción, dirección y los técnicos que desarrollan los efectos digitales y especiales. En una reunión sobre efectos se habla de la luz y de muchos matices que si no se ruedan atendiendo a sus especificaciones, los técnicos de efectos visuales lo tienen muy complicado en postproducción para enriquecer, para añadir o borrar. Nos queda todavía mucho por aprender" (R. Abades, entrevista personal, 26 de junio, 2012).

Pero incluso cuando dentro del equipo de producción existe la figura del productor especializado en postproducción, una parte importante de la creación de efectos visuales se sigue escapando a su control.

"Nos falta conocer, siempre que hablamos de efectos visuales todavía son para muchos "esos locos magos"... este punto que no sabemos muy bien esos tiempos que toman y necesitan los procesos. (...) Aquí lo que falta un poco es también personas que se dediquen y conozcan, el productor de postproducción o el supervisor de efectos visuales, que tiene que entender y conocer los departamentos y hacer de director de producción de toda esta gran familia. Hay mucha formación de gente 
especializada en software, pero falta gente de producción que sepa de lo que está hablando” (J. Martí, entrevista personal, 21 de marzo, 2012).

Para solucionar este problema quizá sea necesaria una colaboración por ambas partes: producción de efectos visuales y el núcleo de producción de la película. Así, por parte del primero, es vital una alta especialización tecnológica que permita entender los factores que condicionarán los costes y tiempos de los distintos efectos visuales que formarán parte de la película. Paralelamente será necesario por parte de los equipos de producción de efectos visuales, la voluntad de compartir información y concretar variables que permitan realizar previsiones fiables, favoreciendo la competitividad y fortaleciendo así el tejido empresarial.

Todo ello implica a su vez la necesidad de abandonar esquemas tradicionalmente asociados a la ausencia de posibilidades narrativas en la fase de postproducción o la falta de potencial de los efectos visuales como herramienta de producción. Esta evolución implica la necesidad de trasladar esta nueva realidad a esquemas productivos que, en muchos casos, parecen seguir anclados en el rodaje como única fase de creación cinematográfica.

\section{Conclusiones}

Los efectos visuales han pasado a formar parte de los procesos productivos cinematográficos en España. Por ello, para poder ejercer un control efectivo de la película, es necesaria una actualización constante por parte de los equipos de producción y una especialización que les permita gestionar de una forma más eficiente una tendencia al crecimiento de la creación cinematográfica en postproducción. Desde nuestro punto de vista, es coherente y necesaria una normalización en la existencia de un productor “experto”26, especializado en postproducción, por tres razones: un elevado factor tecnológico con fuertes implicaciones sobre la eficiencia de los

\footnotetext{
${ }^{26}$ Término utilizado por Jacoste (1996, p. 15) para referirse al profesional de la producción especializado que atesora los conocimientos necesarios sobre los factores que condicionan la temporalización y organización de la producción cinematográfica.
} 
procesos productivos, un aumento de los recursos destinados a la fase de postproducción y la necesidad de mantener un control efectivo sobre la producción de efectos visuales desde el núcleo de producción del film.

Sin embargo en España es habitual la ausencia del productor de efectos visuales y del director de postproducción, cuyas competencias son repartidas entre el personal de oficina de las productoras de efectos visuales, el supervisor de efectos visuales y el núcleo de producción del film. Esta indefinición está provocada por el limitado presupuesto de los proyectos cinematográficos, la falta de solidez empresarial de un sector compuesto por productoras que condicionan su continuidad al éxito de su última película y la falta de agilidad del sector para adaptarse a un escenario productivo en constante transformación. En este sentido, resulta sintomático que el modelo de financiación del ICAA no se haya adaptado desde hace más de quince años y que ni siquiera exista una apartado destinado a especificar el gasto destinado a la producción de efectos visuales digitales y sí de efectos ópticos.

La traslación de parte de la creación visual de la imagen cinematográfica del rodaje a la postproducción genera la necesidad de adaptación a este nuevo contexto por implicar una dilatación de los tiempos de producción, un aumento de profesionales especializados en ese ámbito y la necesidad de gestionar un aumento de los recursos destinados a la creación de efectos visuales. Sin embargo, debemos ser cautos a la hora de valorar esta traslación de recursos, ya que los reducidos costes medios de las películas españolas podrían estar provocando que apenas tengan margen de maniobra después de cubrir las partidas asociadas a la fase de rodaje. En cualquier caso, atendiendo al aumento en la producción de efectos visuales, es necesario el establecimiento de una figura de coordinación de los flujos de trabajo que integran los trabajos de postproducción y que personifique la gestión de los nuevos esquemas productivos. Mientras esto no se produzca, la industria cinematográfica española presentará una debilidad provocada por la inexistencia de una infraestructura organizativa con capacidad para implementar con fiabilidad herramientas digitales en postproducción, favoreciendo la innovación y la competitividad. 
En tiempos difíciles como el actual, en los que la financiación de los proyectos cinematográficos es cada vez más complicada, debemos asumir con la madurez necesaria el proceso de cambio en el que estamos inmersos. Sólo desde la creación de paradigmas de producción ágiles e innovadores, que permitan gestionar correctamente la aplicación de herramientas digitales, los efectos visuales podrán convertirse en un factor dinamizador del cine español como industria cultural.

\section{Referencias bibliográficas}

Billups, S. (2003). Digital moviemaking. Michigan: Michael Wiese Productions.

Bugaj, S. V. (2010). Analysis of a production workflow. En J. Okun, \& S. Zwerman (Eds.), The VES handbook of visual effects (pp. 784-794). Burlington: Elsevier.

Ciller, C. (2009). La producción en la postproducción. El caso de Alatriste. En J. Marzal, y F. Gómez (Eds.), El productor y la producción en la industria cinematográfica (pp. 411-420). Madrid: Editorial Complutense.

De Miguel, R. (2005). La entrevista en profundidad a los emisores y los receptores de los medios. En M. Berganza, \& J. A. Ruiz (Eds.), Investigar en comunicación (pp. 251-263). Madrid: Mc Graw Hill.

Fernández, F., \& Martínez, J. (1994). La dirección de producción para cine y televisión. Barcelona: Paidós.

Finance, C., \& Zwerman, S. (2010). The visual effects producer. Burlington: Elsevier.

Marzal, J. \& Gómez, F. (2009). Introducción. En J. Marzal, \& F. Gómez (Eds.), El productor y la producción en la industria cinematográfica (pp. 11-17). Madrid: Editorial Complutense.

García, R., Constenla, T. \& Verdú, D., "Los recortes encienden la alerta roja”, El País, 27/09/2012.

Gross, L., \& Ward, L. (2004). Digital moviemaking. Belmont: Thomson Wadsworth.

Honthaner, E. L. (2010). The Complete Film Production. Burlington: Elsevier.

Instituto de la Cinematografía y de las Artes Audiovisuales (2011). Boletín informativo: películas, recaudación, espectadores, datos de 2010. 
el

$12 /$ enero/2012

en:

http://www.mcu.es/cine/docs/MC/BIC/2010/Boletin_2010.pdf

Iwerks, L. (Productor y Director) (2007). The pixar history. [Video/DVD] USA: Buena Vista Home entertainment, Walt Disney Studio Motion Pictures.

Jacoste, J. (1996). El productor cinematográfico. Madrid: Síntesis.

Lara, A. (2005). El cine ha muerto, larga vida al cine. Madrid: T \& B.

Lucas, R. C. (2011). Crafting Digital Cinema: Cinematographers in Contemporary Hollywood. [Universidad de Texas, Austin]. Consultado el o8/diciembre/2011 en: http://repositories.lib.utexas.edu/handle/2152/ETD-UT-2011-o84147

Manovich, L. (2001). The language of new media. Massachusetts: MIT Press.

Novy, D. (2010). Virtual studio technology. En J. Okun, y S. Zwerman (Eds.), The VES handbook of visual effects (pp. 783-784). Burlington, USA: Elsevier.

Riambau, E. \& Torreiro, C. (2009). Productores en el cine español: una aproximación histórica. En J. Marzal, \& F. Gómez (Eds.), El productor y la producción en la industria cinematográfica (pp. 81-94). Madrid: Editorial Complutense.

Silverman, L. (2005). The new post production workflow: Today and tomorrow. En Swartz, C. (Ed.) Understanding digital cinema (pp. 1556). Burlington: Elsevier.

Smith, T. G. (1986). Industrial light and magic: The art of special effects. Hong Kong: Ballantine Books.

Spelthann, V. \& Haunschild, A. (2011). Organizational Creativity in Heterarchies: The Case of VFX Production. Creativity and Innovation Management. (20 vol. 2), pp. 100-107.

Swartz, C. (2005). Understanding digital cinema. Burlington: Elsevier.

Towsend, C. (2010). Digital intermediate. En J. Okun, y S. Zwerman (Eds.), The VES hand book of visual effects (pp. 530- 532). Burlington: Elsevier.

Vidal, M. (2008). Contribución de la animación cinematográfica al desarrollo del trucaje cinematográfico y los efectos especiales en el cine contemporáneo (Tesis doctoral), Universidad Politécnica de Valencia, Valencia. Consultada el 2/febrero/2012 en: http://riunet.upv.es/handle/10251/2182 